\title{
Alfvén-Wave Instability of Current Sheets in Force-Free Collisionless Plasmas
}

\author{
P. M. Bellan \\ Caltech, Pasadena, California 91125 \\ (Received 24 May 1999; revised manuscript received 20 September 1999)
}

\begin{abstract}
If the current sheet between adjacent twisted magnetic flux tubes is sufficiently thin, the electron flow velocity becomes comparable to the Alfvén velocity and can destabilize collisionless Alfvén waves. The threshold for instability in force-free plasmas is calculated for both inertial and kinetic Alfvénwave regimes. When there is strong magnetic shear, unstable kinetic Alfvén waves can resonantly accelerate ions to energies much higher than the electron temperature.
\end{abstract}

PACS numbers: 52.35.Qz, 52.35.Hr, 52.35.Py, 52.40.Mj

The direction of the magnetic field rotates abruptly at the interface between two adjacent twisted flux tubes [1] corresponding to a thin current sheet flowing in this interface. The character of the current sheet depends [2] on the ratio of plasma thermal to magnetic energy $\beta=2 \mu_{0} n \kappa T / B^{2}$. When $\beta \sim 1$ (denoted high $\beta$ ), current sheets are associated with a magnetic field null and a localized region of increased plasma pressure $[3,4]$. When $\beta \ll 1$ (denoted low $\beta$ ), the magnetic field magnitude remains approximately constant throughout the current sheet, there is no associated change in plasma pressure, and the plasma is nearly force free.

Low $\beta$ plasmas constitute an important class of plasmas and include the solar corona, the near-Earth magnetosphere, spheromaks, reversed-field pinches, and tokamaks. Magnetic reconnection is experimentally observed in all these plasmas and involves the magnetic field becoming locally unfrozen from the plasma. This has been traditionally modeled by invoking finite Ohmic resistivity to allow magnetic field diffusion and dissipation at the current sheet with consequent modification of the current sheet. However, for many important situations the plasma is essentially collisionless so that resistive models cannot explain magnetic reconnection. There is, consequently, great interest in finding collisionless mechanisms which can modify current sheets and thereby enable magnetic reconnection.

Recently this author [5] proposed that the radiation resistance associated with Alfvén-wave emission by a thin current sheet could act as an effective collisionless resistivity, but the model in Ref. [5] did not explain why a current sheet should spontaneously emit Alfvén waves. This Letter demonstrates that sufficiently thin current sheets in low $\beta$ collisionless plasmas behave as laserlike kinetically unstable resonators which spontaneously emit Alfvén waves.

Low $\beta$ plasmas can be categorized into two subclasses according to whether $\beta$ is smaller or larger than $m_{e} / m_{i}$, and so it is convenient to define a normalized quantity $\bar{\beta}=$ $\beta m_{i} / m_{e}=v_{T e}^{2} / v_{A}^{2}$. The two subclasses have distinct physics [6]: when $\bar{\beta} \ll 1$, Alfvén waves have the inertial Alfvén-wave (IAW) dispersion relation and, when $1 \ll$ $\bar{\beta} \ll m_{i} / m_{e}$, Alfvén waves have the kinetic Alfvén-wave (KAW) dispersion.
Because $\beta \ll 1$, the equilibrium can be approximated as force free (i.e., $\mathbf{J} \times \mathbf{B}=0$ ) so that

$$
\nabla \times \mathbf{B}=\alpha(\mathbf{r}) \mathbf{B} .
$$

The divergence of Eq. (1) shows that $\alpha$ is constant along a field line. Since $J \sim \alpha$, a current sheet corresponds to a sharp peaking of $\alpha$. We construct a coordinate system having $\hat{x}$ parallel to $\nabla \alpha$ and $\hat{z}$ parallel to $\mathbf{B}$ at the current sheet midpoint $x=0$. The magnetic field satisfying Eq. (1) can be expressed in this coordinate system as

$$
\mathbf{B}(x)=\hat{y} B \sin [\theta(x)]+\hat{z} B \cos [\theta(x)],
$$

where $\theta(x)=\int_{0}^{x} \alpha\left(x^{\prime}\right) d x^{\prime}$ gives the rotation of $\mathbf{B}(x)$ relative to its orientation at $x=0$. A convenient representation for $\alpha(x)$ which causes $\mathbf{B}$ to rotate by an angle $\Delta$ in the current sheet is given by

$$
\alpha(x)=\frac{\Delta}{a} \Theta\left(\frac{a}{2}-|x|\right),
$$

where $a$ is the current sheet width and $\Theta$ is the Heaviside function; the corresponding field angle dependence is

$$
\theta(x)= \begin{cases}\frac{x \Delta}{a} & \text { for }|x| \leq a / 2 \\ \frac{\Delta}{2} \operatorname{sgn}(x) & \text { for }|x| \geq a / 2 .\end{cases}
$$

For simplicity, finite Larmor radius effects are neglected by assuming that particles are cold in the direction perpendicular to $\mathbf{B}$. Since the field-aligned current magnitude is $J(x)=\alpha(x) B / \mu_{0}$, the equilibrium electron flow velocity is

$$
u_{\| e 0}(x)=\frac{\alpha(x) B}{\mu_{0} n q}=v_{A} \frac{c \Delta}{\omega_{p i} a} \Theta\left(\frac{a}{2}-|x|\right),
$$

where $v_{A}$ is the Alfvén velocity and terms of order $m_{e} / m_{i}$ have been dropped. Thus if $a \omega_{p i} / c \sim \Delta$, destabilization of Alfvén waves becomes a possibility.

We assume that perturbed quantities vary as $g(x) \exp \left[i k_{\|}(x)_{s}-i \omega t\right]$, where $\omega \ll \omega_{c i}, k_{\|}=k_{z} \cos \theta$, and $s$ is the distance along $\mathbf{B}$. The parallel wave current is 
given by

$$
\begin{aligned}
\tilde{J}_{\|} & =\sum_{\sigma} q_{\sigma} \int d v_{\|} v_{\|} \tilde{f}_{\sigma}\left(v_{\|}\right) \\
& =\frac{i \omega \tilde{E}_{\|}}{\mu_{0} c^{2}} \sum_{\sigma} \frac{1}{2 k_{\|}^{2} \lambda_{D \sigma}^{2}} Z^{\prime}\left(\frac{\omega-k_{\|} u_{\| \sigma 0}}{k_{\|} v_{T \sigma}}\right),
\end{aligned}
$$

where $Z$ is the plasma dispersion function [7] and $v_{T \sigma}=\sqrt{2 \kappa T_{\sigma} / m_{\sigma}}$.

Using the ion polarization drift $\mu_{0} \tilde{\mathbf{J}}_{\perp}=\nabla_{\perp}\left(i k_{\|} \tilde{A}_{\|}\right)=$ $i \omega v_{A}^{-2} \nabla_{\perp} \tilde{\phi}$, the electric field components can be expressed in terms of $\tilde{A}_{\|}$as

$$
\tilde{E}_{\|}=i \omega\left(1-k_{\|}^{2} v_{A}^{2} / \omega^{2}\right) \tilde{A}_{\|}, \quad \tilde{\mathbf{E}}_{\perp}=-\nabla_{\perp}\left(k_{\|} v_{A}^{2} \tilde{A}_{\|} / \omega\right) .
$$

It has been assumed that $\nabla_{\perp} \gg \alpha$ which also means that $\tilde{\mathbf{B}}_{\perp}=\nabla \tilde{A}_{\|} \times \hat{B}_{0}$. Invoking $\mu_{0} \tilde{J}_{\|}=-\nabla_{\perp}^{2} \tilde{A}_{\|}$and substituting for $\tilde{E}_{\|}$in Eq. (6) gives the Alfvén-wave equation

$$
\begin{aligned}
\frac{c^{2}}{\omega_{p e}^{2}} \nabla_{\perp}^{2} \tilde{A}_{\|}+Q(\bar{\beta}, \zeta)[ & Z^{\prime}\left(\zeta-\frac{u_{\| e 0}(x)}{v_{T e}}\right) \\
& \left.+\frac{T_{e}}{T_{i}} Z^{\prime}\left(\zeta \sqrt{\frac{T_{e} m_{i}}{T_{i} m_{e}}}\right)\right] \tilde{A}_{\|}=0,
\end{aligned}
$$

where

$$
Q(\bar{\beta}, \zeta)=\bar{\beta}^{-1}-\zeta^{2},
$$

$\zeta=\omega / k_{\|} v_{T e}$, and ion flow velocity which is of order $m_{e} / m_{i}$ has been dropped. The ion contribution has been retained in Eq. (8) because ion Landau damping can occur for KAW.

Instability in a uniform plasma occurs [8] when the argument of $Z^{\prime}$ becomes negative, i.e., when $u_{\| e 0}>$ $\omega / k_{\|}$so that $\operatorname{Im} Z^{\prime}$ reverses sign. The situation is more complicated for a current sheet because the localization of $u_{\| e 0}$ means there is only a narrow layer where $\operatorname{Im} Z^{\prime}$ reverses sign (beam excitation of purely electrostatic waves $[9,10]$ has a similar localized character).

Physically, Eq. (8) describes spontaneous emission of Alfvén waves by the current sheet; these waves radiate outwards to $x= \pm \infty$ and Landau damp in the region exterior to the current sheet. Global instability results when the rate of wave energy production by the current sheet exceeds the rate at which wave energy is destroyed by Landau damping. Wave emission must come at the expense of the original current sheet and it is expected that quasilinear theory will describe a modification of the current sheet as the waves are radiated. Landau damping of emitted waves on exterior electrons effectively transfers momentum from current sheet electrons to exterior electrons and so acts as an electron viscosity. Landau damping on ions (as might occur for KAW with warm ions and highly sheared fields) transfers momentum from electrons to ions and so acts as a resistivity.

Two boundary conditions apply to the $x$-directed wave energy flux: (i) the wave energy flux vanishes at $x=0$ because of symmetry and (ii) it also vanishes at $x= \pm \infty$ because of exterior region Landau damping. The wave energy flux is proportional to $\operatorname{Im}\left(\tilde{A}_{\|}^{*} d \tilde{A}_{\|} / d x\right)$ and so we derive an equation for this quantity. By assuming $\zeta_{i} \ll \zeta_{r}$ and then Taylor expanding $Q$ and $Z^{\prime}$, Eq. (8) can be written with explicit real and imaginary parts as

$$
\frac{c^{2}}{\omega_{p e}^{2}} \frac{d^{2} \tilde{A}_{\|}}{d x^{2}}+Q\left(\bar{\beta}, \zeta_{r}\right) Z_{R}^{\prime}\left(\bar{\zeta}_{r}\right) \tilde{A}_{\|}+i\left(Q\left(\bar{\beta}, \zeta_{r}\right)\left[Z_{I}^{\prime}\left(\bar{\zeta}_{r}\right)+\frac{T_{e}}{T_{i}} Z_{I}^{\prime}\left(\zeta_{r} \sqrt{\frac{T_{e} m_{i}}{T_{i} m_{e}}}\right)+\zeta_{i} Z_{R}^{\prime \prime}\left(\bar{\zeta}_{r}\right)\right]-2 \zeta_{i} \zeta_{r} Z_{R}^{\prime}\left(\bar{\zeta}_{r}\right)\right) \tilde{A}_{\|}=0
$$

where

$$
\bar{\zeta}_{r}=\zeta_{r}-\frac{u_{\| e 0}(x)}{v_{T e}}=\zeta_{r}-\frac{c}{a \omega_{p i}} \frac{\Delta}{\bar{\beta}^{1 / 2}} \Theta\left(\frac{a}{2}-|x|\right),
$$

and we have noted that the dominant perpendicular derivative is in the $x$ direction. The $\beta \ll 1$ assumption means that $\omega / k_{\|} \boldsymbol{v}_{T i} \gg 1$ for both IAW and KAW; thus the ion term $Z_{R}^{\prime}\left(\zeta_{r} \sqrt{T_{e} m_{i} / T_{i} m_{e}}\right)$ is always much smaller than the corresponding electron term $Z_{R}^{\prime}\left(\zeta_{r}\right)$ and so has been dropped.

Multiplying Eq. (10) by $\tilde{A}_{\|}^{*}$ and then taking the imaginary part of the result yields the wave energy-flux equation

$$
\frac{c^{2}}{\omega_{p e}^{2}} \operatorname{Im}\left[\frac{d}{d x}\left(\tilde{A}_{\|}^{*} \frac{d \tilde{A}_{\|}}{d x}\right)\right]+\left\{Q\left(\bar{\beta}, \zeta_{r}\right)\left[Z_{I}^{\prime}\left(\bar{\zeta}_{r}\right)+\frac{T_{e}}{T_{i}} Z_{I}^{\prime}\left(\zeta_{r} \sqrt{T_{e} m_{i} / T_{i} m_{e}}\right)+\zeta_{i} Z_{R}^{\prime \prime}\left(\bar{\zeta}_{r}\right)\right]-2 \zeta_{i} \zeta_{r} Z_{R}^{\prime}\left(\bar{\zeta}_{r}\right)\right\}\left|\tilde{A}_{\|}\right|^{2}=0
$$

Integrating Eq. (12) from $x=0$ to $x=\infty$ and invoking boundary conditions (i) and (ii) gives an equation linear in $\omega_{i}=\zeta_{i} k_{\|} v_{T e}$ which may be solved to give

$$
\omega_{i}=-\frac{\int_{0}^{\infty} d x\left|\tilde{A}_{\|}\right|^{2} Q\left(\bar{\beta}, \zeta_{r}\right)\left[Z_{I}^{\prime}\left(\bar{\zeta}_{r}\right)+\frac{T_{e}}{T_{i}} Z_{I}^{\prime}\left(\zeta_{r} \sqrt{\frac{T_{e} m_{i}}{T_{i} m_{e}}}\right)\right]}{\int_{0}^{\infty} d x \frac{\left|\tilde{A}_{\|}\right|^{2}}{k_{\|} v_{T e}}\left[Q\left(\bar{\beta}, \zeta_{r}\right) Z_{R}^{\prime \prime}\left(\bar{\zeta}_{r}\right)-2 \zeta_{r} Z_{R}^{\prime}\left(\bar{\zeta}_{r}\right)\right]} .
$$


The integrals in Eq. (13) are convergent because Landau damping in the exterior region causes $\left|\tilde{A}_{\|}\right|^{2}$ to decay exponentially at large $x$. Equation (13) is a generalization of the standard result that Taylor expansion of the uniform plasma dispersion relation $D\left(\omega_{r}+i \omega_{i}\right)=0$ gives $\left[\omega_{i}\right]_{\text {uniform }}=-D_{I} /\left[\partial D_{R} / \partial \omega_{r}\right]$.

Evaluation of Eq. (13) is nontrivial because the exterior region decay rate of $\left|\tilde{A}_{\|}\right|^{2}$ depends on $\omega_{i}$. This complication is avoided here by restricting the analysis to a search for marginal stability, i.e., the condition that $\omega_{i}$ changes from negative to positive.

The real and imaginary parts of Eq. (10) constitute two coupled second order ordinary differential equations with two-point boundary conditions. We have solved this system numerically for marginal instability using the method in Ref. [11] with $Z^{\prime}$ prescribed by the two-pole approximation [7]. In addition to the energy-flux boundary condition that $\operatorname{Im}\left(\tilde{A}_{\|}^{*} d \tilde{A}_{\|} / d x\right)$ vanishes at $x=0, \pm \infty$ it is also necessary for $\tilde{A}_{\|}$to be an even function of $x$ to have continuity of both $\tilde{A}_{\|}$and $d \tilde{A}_{\|} / d x$ at $x=$ 0 . This parity condition provides a laserlike quantization condition on allowed $a, \zeta_{r}^{\text {ext }}$ combinations and corresponds to requiring a smooth matching of the current sheet $\tilde{A}_{\|} \sim \exp \left(i k_{x}^{\text {sheet }} x\right)+\exp \left(-i k_{x}^{\text {sheet }} x\right)$ to the exterior $\tilde{A}_{\|} \sim \exp \left(i k_{x}^{\text {ext }}|x|\right)$; note that $\operatorname{Im} k_{x}^{\text {sheet }}<0$, whereas $\operatorname{Im} k_{x}^{\text {ext }}>0$. Marginal instability for given $\Delta$ and $\bar{\beta}$ is found by adjusting $a$ and $\zeta_{r}^{\text {ext }}$ until the numerically evaluated numerator of Eq. (13) vanishes while also satisfying $\operatorname{Re} d \tilde{A}_{\|} / d x=\operatorname{Im} d \tilde{A}_{\|} / d x=0$ at $x=0$.

Figure 1 plots numerical integration results for a typical KAW. Here, $\bar{\beta}=200$ and $\Delta=0.87 \pi$ were specified and then $\zeta_{r}^{\text {ext }}$ and $a$ were adjusted to obtain a marginally unstable wave, i.e., a numerical solution of Eq. (10) having $\zeta_{i}=0$ and satisfying all the criteria listed in the previous paragraph. For this case it was found that marginal instability occurs when $\zeta_{r}^{\text {ext }}=0.62$ and $a=0.33 c / \omega_{p i}$. The top three curves in Fig. 1 are, respectively, $\bar{\zeta}_{r}(x)$ [from Eq. (11)], the numerically integrated $\operatorname{Re} \tilde{A}_{\|}$and $\operatorname{Im} \tilde{A}_{\|}$, and $\left|\tilde{A}_{\|}\right|^{2}$. The next curve (marked "Net Power Dissipation") shows the integrand of the numerator of Eq. (13), i.e., $\left|\tilde{A}_{\|}\right|^{2} Q\left(\bar{\beta}, \zeta_{r}\right)\left[Z_{I}^{\prime}\left(\bar{\zeta}_{r}\right)+Z_{I}^{\prime}\left(\zeta_{r} \sqrt{T_{e} m_{i} / T_{i} m_{e}}\right) T_{e} / T_{i}\right]$, and clearly shows how wave emission in the current sheet is balanced by wave damping in the exterior region.

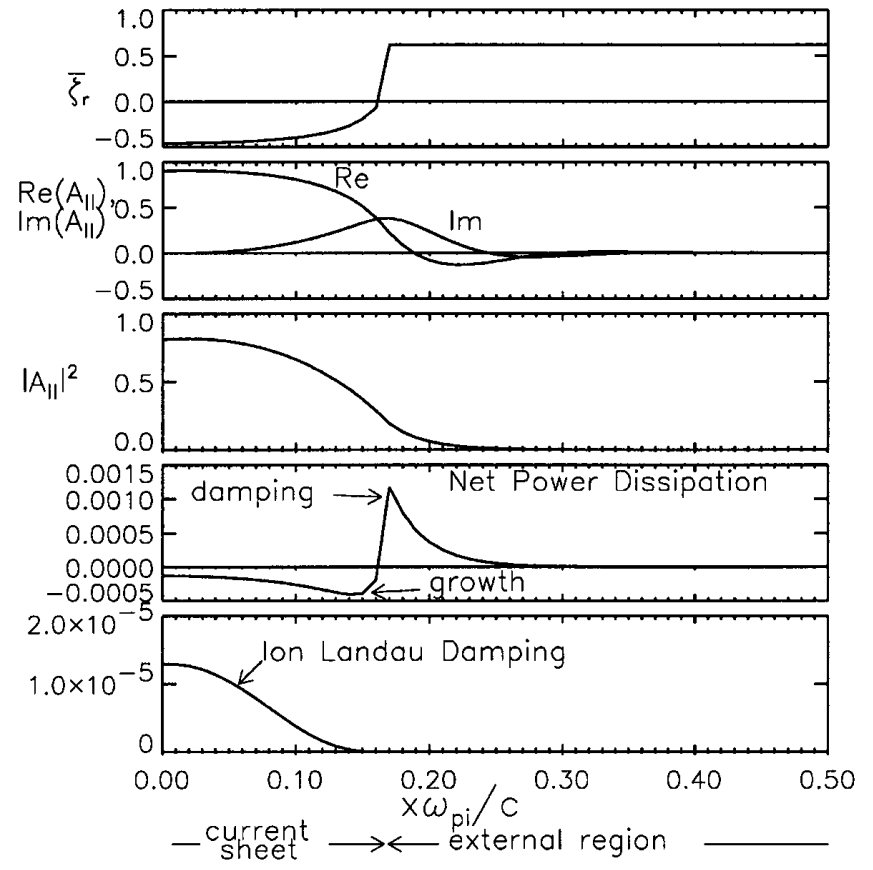

FIG. 1. Numerically calculated wave functions demonstrating a marginally unstable KAW wave (parameters given in text); all plotted quantities are symmetric about $x=0$.

The bottom curve in Fig. 1 shows the ion Landau damping term $\left|\tilde{A}_{\|}\right|^{2} Q\left(\bar{\beta}, \zeta_{r}\right) Z_{I}^{\prime}\left(\zeta_{r} \sqrt{T_{e} m_{i} / T_{i} m_{e}}\right) T_{e} / T_{i}$. Since $k_{\|}=k_{z} \cos \theta$ and $k_{z}$ is invariant, $\zeta_{r}^{\text {sheet }}=$ $\zeta_{r}^{\text {ext }} \cos (\Delta / 2) / \cos [\theta(x)]$ is smaller in the current sheet than in the external region causing ion Landau damping to be concentrated in the current sheet. For KAW which already have small $\zeta_{r}^{\text {ext }}$, the $x$ dependence of $\zeta_{r}$ can result in the wave phase velocity in the current sheet being reduced to a small multiple of $\boldsymbol{v}_{T i}$ and so give significant wave-ion coupling. The $x$ dependence of $\zeta_{r}$ can also cause $Q^{\text {sheet }}$ to differ substantially from $Q^{\text {ext }}$ if $\Delta$ approaches $\pi$.

An analytic estimate of the marginal instability criterion can be obtained by using the approximations $\lim _{\zeta \gg 1} Z^{\prime}(\zeta)=\zeta^{-2}-2 i \pi^{1 / 2} \zeta \exp \left(-\zeta^{2}\right)$ and $\lim _{\zeta \ll 1} Z^{\prime}(\zeta)=-2-2 i \pi^{1 / 2} \zeta \exp \left(-\zeta^{2}\right)$ to find the conditions under which the numerator in Eq. (13) vanishes. It is convenient to split this numerator into the current sheet and exterior regions giving

$$
\int_{0}^{a / 2} d x\left|\tilde{A}_{\|}\right|^{2} Q\left(\bar{\beta}, \zeta_{r}\right)\left[Z_{I}^{\prime}\left(\zeta_{r}-\frac{c}{a \omega_{p i}} \frac{\Delta}{\bar{\beta}^{1 / 2}}\right)+\frac{T_{e}}{T_{i}} Z_{I}^{\prime}\left(\zeta_{r} \sqrt{T_{e} m_{i} / T_{i} m_{e}}\right)\right]+Q\left(\bar{\beta}, \zeta_{r}^{\mathrm{ext}}\right) Z_{I}^{\prime}\left(\zeta_{r}^{\mathrm{ext}}\right) \int_{a / 2}^{\infty}\left|\tilde{A}_{\|}\right|^{2} d x=0 .
$$

External region ion Landau damping has been dropped since it is negligible.

Because the exterior region plasma is uniform, we may assume that in this region $\tilde{A}_{\|} \sim \exp \left(i k_{x} x\right)$, where $k_{x}=$ $k_{x r}+i k_{x i}$. The real part of Eq. (10) therefore becomes the dispersion relation [6]

$$
k_{x r}= \pm \frac{\omega_{p e}}{c} \sqrt{Q\left(\bar{\beta}, \zeta_{r}\right) Z_{R}^{\prime}\left(\zeta_{r}\right)}
$$

If $\bar{\beta} \ll 1$, Eq. (15) becomes the IAW dispersion $k_{x r}=-\left(k_{\|}^{2} v_{A}^{2} / \omega_{r}^{2}-1\right)^{1 / 2} \omega_{p e} / c$ and has propagating waves when $\bar{\beta}^{-1 / 2}>\zeta_{r}>1$, whereas if $1 \ll$ $\bar{\beta} \ll m_{i} / m_{e}$ Eq. (15) becomes the KAW dispersion $k_{x r}=+\left(\omega_{r}^{2}-k_{\|}^{2} v_{A}^{2}\right)^{1 / 2} / c k_{\|} \lambda_{D e}$ with propagating waves when $\bar{\beta}^{-1 / 2}<\zeta_{r}<1$. For IAW both $Q$ and $Z_{R}^{\prime}$ are positive while for KAW both are negative. In order 
to have outward wave energy propagation for $x>0$, we have chosen $k_{x r}$ negative for IAW (backwards wave) and positive for KAW (forwards wave).

Because $\omega_{i}=0$ at marginal instability, the imaginary part of the exterior region dispersion relation associated with Eq. (10) yields

$$
k_{x i}^{\mathrm{ext}}=\frac{\omega_{p e} \pi^{1 / 2}}{c} \sqrt{\frac{Q\left(\bar{\beta}, \zeta_{r}\right)}{Z_{R}^{\prime}\left(\zeta_{r}\right)}} \zeta_{r} \exp \left(-\zeta_{r}^{2}\right) .
$$

$\left|\tilde{A}_{\|}\right|^{2}$ may be approximated as uniform within the thin current sheet, and so in the current sheet $\left|\tilde{A}_{\|}\right|^{2} \approx\left|\tilde{A}_{\|}(0)\right|^{2}$. However, in the external region the wave energy decays as $\left|\tilde{A}_{\|}(x)\right|^{2}=\left|\tilde{A}_{\|}(0)\right|^{2} \exp \left(-2 k_{x i}^{\text {ext }} x\right)$ and so

$$
\int_{a / 2}^{\infty}\left|\tilde{A}_{\|}\right|^{2} d x=\frac{\left|\tilde{A}_{\|}(0)\right|^{2}}{2 k_{x i}^{\mathrm{ext}}} \exp \left(-k_{x i}^{\mathrm{ext}} a\right) .
$$

Combining Eqs. (14) and (17) gives the condition for marginal instability to be

$$
\begin{aligned}
{\left[Q Z_{I}^{\prime}\left(\zeta_{r}-\frac{c}{a \omega_{p i}} \frac{\Delta}{\bar{\beta}^{1 / 2}}\right)\right.} & \left.+Q \frac{T_{e}}{T_{i}} Z_{I}^{\prime}\left(\zeta_{r} \sqrt{\frac{T_{e} m_{i}}{T_{i} m_{e}}}\right)\right]_{\text {sheet }} \\
& >\frac{2 c}{a \omega_{p i}} \sqrt{\frac{m_{e}}{m_{i}}} \\
& \times\left[\sqrt{Q Z_{R}^{\prime}\left(\zeta_{r}\right)} e^{-k_{x i} a}\right]_{\mathrm{ext}}
\end{aligned}
$$

or, in a more convenient dimensionless form

$$
\begin{aligned}
{\left[\left(\mathrm{Y}-\zeta_{r}\right) e^{-\left(\mathrm{Y}-\zeta_{r}\right]^{2}}\right]_{\text {sheet }} } & >\left[\zeta_{r} I\right]_{\text {sheet }} \\
& +\frac{Q^{\text {ext }}}{Q^{\text {sheet }}}\left[\Upsilon \varepsilon \exp \left(-\frac{e^{-\zeta_{r}^{2}}}{\varepsilon}\right)\right]_{\text {ext }},
\end{aligned}
$$

where $\mathrm{Y}=c \Delta / a \omega_{p i} \bar{\beta}^{1 / 2}$ is the normalized inverse current sheet thickness, $I=\left(T_{e} / T_{i}\right)^{3 / 2}\left(m_{i} / m_{e}\right)^{1 / 2}$ $\exp \left(-\zeta_{r}^{2} T_{e} m_{i} / T_{i} m_{e}\right)$ characterizes ion Landau damping, and $\varepsilon=\left[\sqrt{\bar{\beta} m_{e} Z_{R}^{\prime}\left(\zeta_{r}\right) / \pi \Delta^{2} m_{i} Q\left(\bar{\beta}, \zeta_{r}\right)}\right]_{\mathrm{ext}}$ characterizes exterior region electron Landau damping.

Equation (19) is a function of two undetermined dimensionless variables, $Y$ and $\zeta_{r}^{\text {ext }}$; all other quantities are set by the equilibrium. Two necessary conditions for instability are the following: (i) $\mathrm{Y}>\zeta_{r}^{\text {sheet }}$ which indicates that instability begins only when $a$ becomes sufficiently small; (ii) $\varepsilon<1$ which gives a shear requirement $\Delta>\left[\sqrt{\bar{\beta} m_{e} Z_{R}^{\prime}\left(\zeta_{r}\right) / \pi m_{i} Q\left(\bar{\beta}, \zeta_{r}\right)}\right]_{\mathrm{ext}}$.

Equation (19) indicates that, when $a$ decreases monotonically (e.g., when two flux tubes collide), there will be a narrow, but finite, $a$ range giving instability. It must be recalled that the matching conditions on $\tilde{A}_{\|}$and $d \tilde{A}_{\|} / d x$ quantize allowed values of $a$. For example, consider the IAW example of a hydrogen plasma with $\bar{\beta}=0.14$ and $\Delta=0.01 \pi$. Numerical integration using the two-pole $Z^{\prime}$ representation and including quantization shows that the combination $\zeta_{r}^{\text {ext }}=2.56$ and $a \omega_{p i} / c=0.024$ gives marginal instability. For comparison, using $\zeta_{r}^{\text {ext }}=2.56$ in Eq. (19) predicts a range $0.022<a \omega_{p i} / c<0.030$ for IAW instability showing that Eq. (19) provides a reasonable estimate of the instability threshold.

When $\Delta$ is large and the plasma is in the KAW regime, the wave in the current sheet can be sufficiently slowed to resonate with tail ions (cf. bottom curve in Fig. 1). Thus, when $\Delta$ is large, KAW instability can accelerate current sheet ions to energies $\gg T_{e}$. This linear analysis predicts interaction with only the small number of tail ions, but, if a tail-pulling effect analogous to that observed in lower-hybrid current drive occurs, a much more intense ion tail would be produced. This KAW acceleration of current sheet ions is a likely explanation for energetic ion beams observed by Kornack et al. [12] in the plane of the reconnection layer between two merging spheromaks; if tail pulling occurs, it could also explain the efficient generation of hot ions observed by Ono et al. [13] in the TS-3 merging spheromak reconnection experiment.

The author wishes to thank the International Space Science Institute (Bern, Switzerland) for hosting a series of Alfvén-wave workshops which stimulated thought on this topic. This work was supported by USDOE.

[1] E. N. Parker, Astrophys. J. 264, 635 (1983).

[2] M. Yamada et al., Phys. Plasmas 4, 1936 (1997).

[3] M. A. Shay et al., J. Geophys. Res. 103, 9165 (1998).

[4] W. Daughton, Phys. Plasmas 6, 1329 (1999), and references therein.

[5] P. M. Bellan, Phys. Plasmas 5, 3081 (1998).

[6] D. Leneman et al., Phys. Rev. Lett. 82, 2673 (1999), and references therein.

[7] B.D. Fried and S.D. Conte, The Plasma Dispersion Function (Academic Press, New York, 1961).

[8] D. D. Meyerhofer and F. W. Perkins, Phys. Fluids 27, 2483 (1984).

[9] L. D. Pearlstein and H.L. Berk, Phys. Rev. Lett. 23, 220 (1969).

[10] G. Ganguli and P. Bakshi, Phys. Fluids 25, 1830 (1982); P. Bakshi et al., Phys. Fluids 26, 1808 (1983); G. Ganguli et al., Phys. Fluids 27, 2039 (1984); K. J. Reitzel and G. J. Morales, Phys. Plasmas 5, 3806 (1998).

[11] P. M. Bellan, J. Comput. Phys. 136, 654 (1997).

[12] T. W. Kornack et al., Phys. Rev. E 58, R36 (1998).

[13] Y. Ono et al., Phys. Rev. Lett. 76, 3328 (1996). 